\title{
Eclipse of the B3V companion and flaring of emission lines in V838 Monocerotis
}

\author{
U. Munari ${ }^{1}$, R. L. M. Corradi ${ }^{2,3}$, A. Henden ${ }^{4}$, H. Navasardyan ${ }^{1}$, M. Valentini ${ }^{1}$, R. Greimel ${ }^{2}$, P. Leisy ${ }^{2,3}$, T. Augusteijn ${ }^{5}$, \\ A. A. Djupvik ${ }^{5}$, L. Glowienka ${ }^{5}$, A. Somero ${ }^{5}$, I. G. de la Rosa ${ }^{3}$, A. Vazdekis ${ }^{3}$, I. Kolka ${ }^{6}$, and T. Liimets ${ }^{6}$ \\ 1 INAF - Osservatorio Astronomico di Padova, via dell'Osservatorio 8, 36012 Asiago (VI), Italy \\ e-mail: ulisse.munari@oapd.inaf.it \\ 2 Isaac Newton Group of Telescopes, Apartado de Correos 321, 38700 Sta. Cruz de La Palma, Spain \\ 3 Instituto de Astrofisica de Canarias, 38205 La Laguna, Tenerife, Spain \\ 4 American Association of Variable Star Observers, 49 Bay State Road, Cambridge, MA 02138, USA \\ 5 Nordic Optical Telescope, Apartado de Correos 474, 38700 Sta. Cruz de La Palma, Spain \\ 6 Tartu Observatory, Toravere 61602, Estonia
}

Received 10 May 2007 / Accepted 11 July 2007

\begin{abstract}
After four years during which only the spectacular light echo was showing continuous and rapid evolution while the central star was nearly constant, in autumn 2006 V838 Mon began a sequence of events which profoundly altered its spectroscopic and photometric behavior: $(a)$ an eclipse of the $\mathrm{B} 3 \mathrm{~V}$ companion, characterized by the disappearance and reappearance of the $\mathrm{B} 3 \mathrm{~V}$ companion from optical spectra, and an eclipse-like lightcurve of $\sim 70$ day duration and $\Delta B \sim 1.15 \mathrm{mag}, \Delta V \sim 0.55 \mathrm{mag}, \Delta R_{\mathrm{C}} \sim 0.10$ mag maximum depth; $(b)$ a large increase in intensity of the [FeII] and FeII emission lines, and the appearance in emission for the first time since the 2002 outburst of $\mathrm{H} \alpha$ and higher Balmer series lines. While the [FeII] and FeII lines maintained a very sharp and unresolved profile, the $\mathrm{H} \alpha$ developed into a wide and structured profile, characterized by a sharp central reversal at the same velocity as one of the $\mathrm{CO}$ radio emission components. The disappearance of the $\mathrm{B} 3 \mathrm{~V}$ companion is equally well explained by a grazing eclipse from the outbursting L-type supergiant or by an eclipse from a dust cloud characterized by $E_{B-V}=0.55$ and $R_{V}=3.1$. We believe the flaring of the emission lines occurred at a similar time as the B3V eclipse just by chance.
\end{abstract}

Key words. stars: individual: V838 Monocerotis - stars: winds, outflows - stars: emission-line, Be - binaries: eclipsing

\section{Introduction}

V838 Mon is no doubt one of the most fascinating and studied individual objects of the last few years. Discovered in outburst on January 6, 2002 (Brown 2002), it soon attracted interest for its spectrum characterized by a cool, extended, optically thick and expanding atmosphere, with a complex pattern of metallic lines in absorption, some displaying P-Cyg profiles of several hundred $\mathrm{km} \mathrm{s}^{-1}$ terminal velocity (Munari et al. 2002). The much brighter second maximum reached in early February changed the spectral appearance to that of an early A-type giant with a large flare-up of hydrogen emission lines, while ionized metals with P-Cyg profiles replaced neutral ones. Shortly after that, we discovered the emergence of a light echo around V838 Mon, the first in several decades in the Galaxy (Henden et al. 2002). The light echo progressively increased in size and brightness to become easily observable even with backyard telescopes, while the Hubble Space Telescope started following, and it is still tracking, the details of its spectacular evolution (Bond et al. 2003, 2007).

* Tables 2-4 are only available in electronic form at http: //www. aanda.org

$\star \star$ Based on observations made with the Asiago $1.82 \mathrm{~m}$ of the INAF Astronomical Observatory of Padova, and with $4.2 \mathrm{~m} \mathrm{WHT}$ and $2.5 \mathrm{~m}$ INT operated by the Isaac Newton Group of Telescopes, and with the $2.6 \mathrm{~m}$ NOT operated jointly by Denmark, Finland, Iceland, Norway, and Sweden, in the Spanish Observatorio del Roque de Los Muchachos of the Instituto de Astrofísica de Canarias.
At the beginning of April 2002, V838 Mon went through a rapid drop in optical brightness $(\Delta B=6.5 \mathrm{mag})$ caused by a rapid cooling of the photospheric temperature that, in just one month, caused the spectra to sweep through the entire sequence of $\mathrm{M}$ giant spectral types and beyond, venturing into the temperature regime of L-type brown dwarfs while retaining supergiant dimensions. Balmer and all other emission lines switched off and minimum surface temperature was reached around January 2003 , when the radius was $R \sim 6000 R_{\odot}$ and $T_{\text {eff }} \sim 2000 \mathrm{~K}$ (Pavlenko et al. 2007). At the same time, the surface cooling (and thus shifting of the emission peak of the outbursting component to the IR) allowed the discovery at $U$ and $B$ wavelengths of a B3V companion (Desidera \& Munari 2002). Since January 2003 the outbursting component has entered a very slow and monotonic increase in photospheric temperature so that its optical colors and molecular spectrum in mid-2006 resembled those of the coolest known M-type super-giants.

A recent conference was devoted entirely to V838 Mon (Corradi \& Munari 2007), with detailed reviews of the photometric (Henden 2007) and spectroscopic evolution (Munari et al. 2007) until summer 2006. In this paper we report the large and completely unanticipated photometric and spectroscopic changes that occurred in V838 Mon at the end of 2006, after four years of remarkable stability and that add further complexity to this intriguing and extremely peculiar object. It deserves continuous monitoring at all wavelengths given its inclination to offer continued surprises to its devoted observers. 
There are at least two other known objects with spectroscopic and photometric evolution very similar to that of V838 Mon. M 31-RV (Rich et al. 1989; Mould et al. 1990; Boschi \& Munari 2004) erupted in the bulge of the Andromeda Galaxy (M 31) in 1988, developed an M-type supergiant spectrum at optical maximum with pronounced P-Cyg profiles and Balmer lines in emission, and peaked to $M_{V}=-9.95$. V4332 Sgr (Martini et al. 1999; Munari et al. 2007) erupted in 1994 in the Bulge of our galaxy, peaked at $M_{R c} \sim-9$, and it too developed at optical maximum an M-type supergiant spectrum with Balmer and FeII $+[\mathrm{FeII}]$ emission lines. For both M 31-RV and V4332 Sgr, the observations stopped at the time when the objects were rapidly sweeping through the M-type supergiant spectral sequence (cf. Munari et al. 2007, their Fig. 8), so it is not known if they too ended up developing an L-type supergiant spectrum.

Kimeswenger (2007) has proposed that V1148 Sgr could also be related to V838 Mon type of objects. Discovered as Nova Sgr 1943, it was observed by Mayall (1949) to develop K- and M-type absorption spectra. Not much else is known about this object. Kulkarni et al. (2007) has recently described a bright optical transient source (named M 85 OT2006-1) which appeared in early 2006 in the lenticular galaxy M 85 in the Virgo cluster. It peaked at $M_{R c} \sim-12$, and developed Balmer emission lines on top of a cool spectral energy distribution $\left(T_{\text {eff }} \approx 4600 \mathrm{~K}\right)$. The analogy with V838 Mon is probably even deeper. In fact, looking in detail at Fig. 3 of Kulkarni et al. (2007) which zooms onto a small portion around $\mathrm{H} \alpha$ of their Keck LRIS spectrum for February 24.59, 2006 UT, a deep and wide P-Cyg line profile appears at the (red-shifted) position of BaII $6497 \AA$. BaII lines displayed spectacular P-Cyg profiles in V838 Mon too (cf. Fig. 4 of Munari et al. 2007), especially at the time its continuum mimicked an early K-type supergiant of a temperature similar to that derived by Kulkarni et al. (2007) for M 85 OT2006-1. Kato (2003) discussed CK Vul, which erupted in 1670, in relation to V838 Mon. The association with V838 Mon however could be spurious according to van Hoof et al. (2006) and Kimeswenger (2007), who both prefer a late or very late thermal pulse interpretation for this object. A detailed, multi-frequency investigation by Hajduk et al. (2007) highlights a complex structure in CK Vul revealing at its center what seems to be the remnant of a circumbinary disk, as seen in some binary post-AGB stars.

A wide range of possible explanations have been offered to account for V838 Mon-type eruptions. Iben \& Tutukov (1992) modeled M31-RV in terms of a cool WD accreting at a very low rate from a companion, and under such circumstances the entire WD should have experienced a thermonuclear runaway. To account for V838 Mon, Soaker \& Tylenda (2003, see also Tylenda $\&$ Soker 2006) proposed a stellar merger in a $8+0.3 M_{\odot}$ binary system, while Retter \& Marom (2003, see also Retter et al. 2006) opted for an expanding giant which swallowed orbiting planets. Lawlor $(2005,2007)$ discussed a model for the outburst of V838 Mon based on a born-again plus accretion scenario on a $1 M_{\odot}$ star in a binary system. Munari et al. (2005) suggested for V838 Mon a helium flash on a once more massive progenitor, evolved by heavy mass-loss into the region of Wolf-Rayet stars at the time of observed eruption. Goranskij et al. (2007) similarly argued in favor of a hot and massive progenitor binary system for V4332 Sgr.

\section{Observations}

CCD photometry has been obtained with the Sonoita Research Observatory $0.35-\mathrm{m}$ robotic telescope, using $B V R_{\mathrm{C}} I_{\mathrm{C}}$ filters and an SBIG STL-1001E CCD camera. Pixel size is $1.25^{\prime \prime} /$ pix and
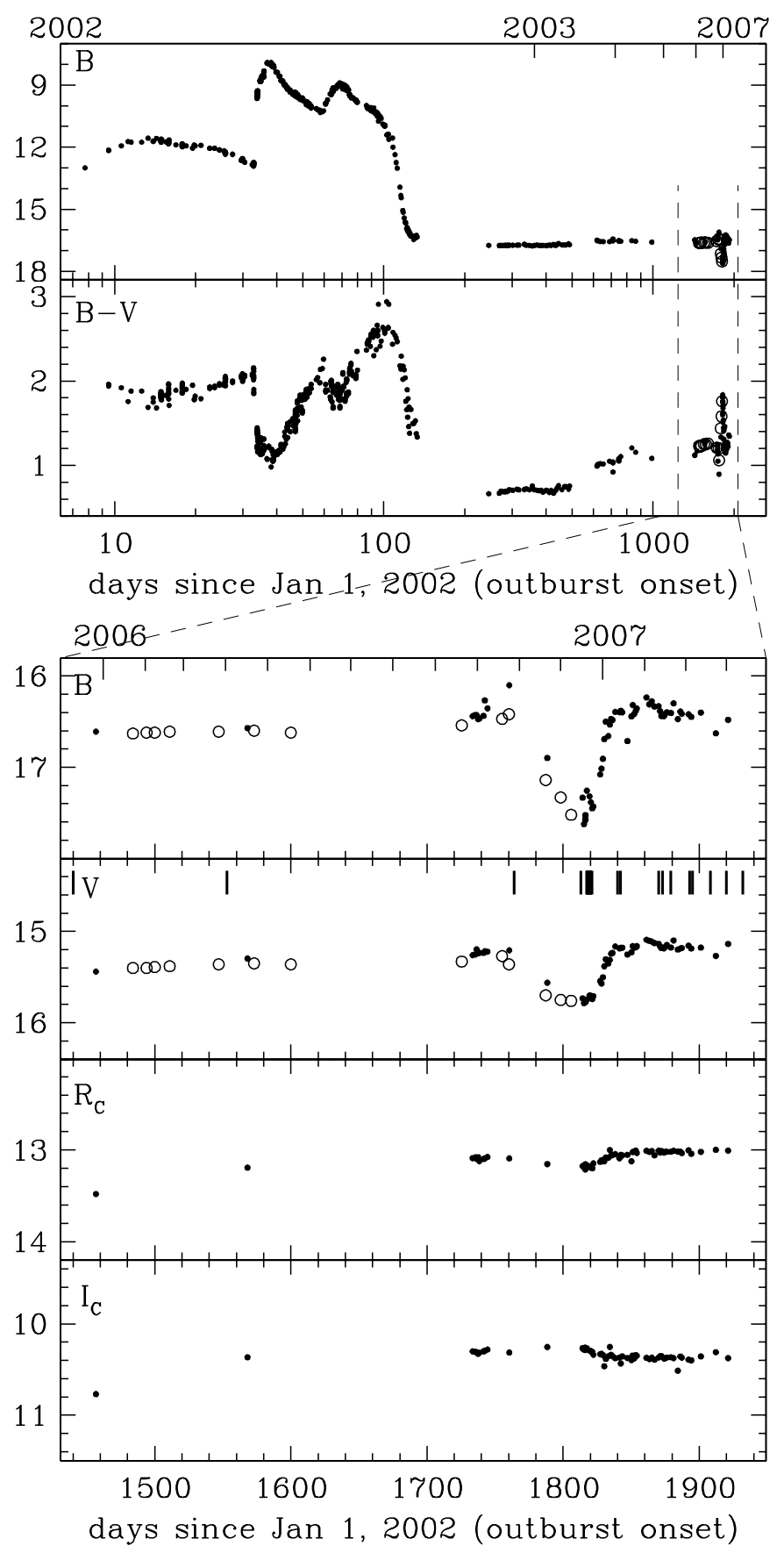

Fig. 1. Upper panels: $B$ and $B-V$ evolution of V838 Mon since outburst onset (data from Henden 2007 and Table 2). Lower panels: zooming on the evolution during the last 16 months. The vertical bars on the $V$ panel mark the epochs of the spectra listed in Table 1. The filled circles are our observations from Table 2, open circles represent observations by Goranskij (2006) and Bond (2006).

the field of view is $20 \times 20^{\prime}$. Exposure times were $8 \times 300 \mathrm{~s}$ in $B, 4 \times 240 \mathrm{~s}$ in $V, 4 \times 120 \mathrm{~s}$ in $R_{\mathrm{C}}$ and $4 \times 60 \mathrm{~s}$ in $I_{\mathrm{C}}$. PSF fitting was adopted instead of aperture photometry, due to the proximity of similar-brightness companion stars. The photometric data are presented in Table 2 (available electronically only) and plotted in Fig. 1.

Spectroscopic observations of V838 Mon were obtained with a number of instruments and a journal of the observations is given in Table 1. Low and medium resolution, absolutely fluxed spectra of V838 Mon have been regularly secured since the 
Table 1. Journal of our spectroscopic observations of V838 Mon.

\begin{tabular}{|c|c|c|c|c|}
\hline date & range $(\AA)$ & $\begin{array}{l}\text { disp. } \\
(\AA / \text { pix })\end{array}$ & $\begin{array}{c}\text { resol. } \\
(\AA)\end{array}$ & instrument \\
\hline 2006 Apr 02 & $\begin{array}{l}3910-7793 \\
8269-9167\end{array}$ & $\begin{array}{l}4.25 \\
0.88\end{array}$ & $\begin{array}{l}10.5 \\
2.42\end{array}$ & Asiago $1.8 \mathrm{~m}+\mathrm{AFOSC}$ \\
\hline Oct 30 & $\begin{array}{l}3948-5954 \\
6030-9128\end{array}$ & $\begin{array}{l}1.61 \\
1.61\end{array}$ & $\begin{array}{l}4.90 \\
5.10\end{array}$ & WHT + INTEGRAL \\
\hline Dec 18 & $3856-5544$ & 0.44 & 1.70 & WHT $4.2 \mathrm{~m}+$ ISIS \\
\hline 22 & $\begin{array}{l}3880-7796 \\
6348-7078\end{array}$ & $\begin{array}{l}4.25 \\
0.72\end{array}$ & $\begin{array}{l}10.5 \\
2.00\end{array}$ & Asiago $1.8 \mathrm{~m},+\mathrm{AFOSC}$ \\
\hline & $8269-9167$ & 0.88 & 2.42 & $"$ \\
\hline 23 & $6378-6682$ & 0.26 & 0.65 & NOT $2.6 \mathrm{~m}+$ ALFOSC \\
\hline 24 & $6378-6682$ & 0.26 & 0.65 & \\
\hline 25 & $6378-6682$ & 0.26 & 0.65 & \\
\hline 26 & $\begin{array}{l}3945-4723 \\
5862-6637\end{array}$ & $\begin{array}{l}0.22 \\
0.25\end{array}$ & $\begin{array}{l}0.75 \\
0.68\end{array}$ & WHT $\underset{\#}{4.2 \mathrm{~m}}+$ ISIS \\
\hline 31 & $6378-6682$ & 0.26 & 0.65 & NOT $2.6 \mathrm{~m}+$ ALFOSC \\
\hline 2007 Jan 14 & $6204-7248$ & 0.47 & 0.98 & INT $2.5 \mathrm{~m}+$ IDS \\
\hline 16 & $6204-7248$ & 0.47 & 0.98 & . \\
\hline Feb 13 & $3500-7800$ & 4.25 & 10.5 & Asiago $1.8 \mathrm{~m}+\mathrm{AFOSC}$ \\
\hline & $6348-7078$ & 0.72 & 2.00 & \\
\hline & $8269-9167$ & 0.88 & 2.42 & $"$ \\
\hline 16 & $3210-5190$ & 1.93 & 4.25 & Asiago $1.8 \mathrm{~m}+\mathrm{AFOSC}$ \\
\hline 22 & $6378-6682$ & 0.26 & 0.65 & NOT $2.6 \mathrm{~m}+$ ALFOSC \\
\hline Mar 10 & $3500-7800$ & 4.25 & 10.5 & Asiago $1.8 \mathrm{~m}+\mathrm{AFOSC}$ \\
\hline & $6348-7078$ & 0.72 & 2.00 & \\
\hline 23 & $3210-5190$ & 1.93 & 4.25 & Asiago $1.8 \mathrm{~m}+$ AFOSC \\
\hline Apr 04 & $6378-6682$ & 0.26 & 0.65 & NOT $2.6 \mathrm{~m}+$ ALFOSC \\
\hline 16 & $3210-5190$ & 1.93 & 4.25 & Asiago $1.8 \mathrm{~m}+$ AFOSC \\
\hline 20 & $5000-7800$ & 4.25 & 10.5 & Asiago $1.8 \mathrm{~m}+\mathrm{AFOSC}$ \\
\hline
\end{tabular}

outburst in January 2002 with the AFOSC imager+spectrograph mounted on the $1.82 \mathrm{~m}$ telescope operated in Asiago by INAF Astronomical Observatory of Padova. Spectra of V838 Mon were collected also with several telescopes of the Observatorio del Roque de los Muchachos (La Palma, Spain).

\section{Eclipse of the B3V companion}

After four years of extremely quiet and flat behaviour (Fig. 1) suddenly, in late October 2006, V838 Mon began a steep decline in brightness which reached a minimum around mid-December and returned to normal brightness by early January 2007. The amplitude of the event was $\Delta B \sim 1.15, \Delta V \sim 0.55$, and $\Delta R_{\mathrm{C}} \sim 0.10 \mathrm{mag}$. We interpret the event as an eclipse of the $\mathrm{B} 3 \mathrm{~V}$ companion. In fact, during the eclipse the signatures of the B3V companion disappeared from the spectra, as clearly shown in Fig. 3, to reappear as soon as the system had regained normal brightness.

In Fig. 2, the spectrum for December 22, obtained close to minimum, shows deeper than usual molecular absorptions from the L-type supergiant (hereafter LtSG). In fact, such molecular absorptions reach zero intensity in their cores, and outside the eclipse they are partially filled-in by the emission from the B3V companion. The apparently deeper molecular absorptions in December do not trace photospheric changes in the LtSG but instead confirm the eclipse scenario and account well for the observed $\Delta R_{\mathrm{C}}=0.10 \mathrm{mag}$ depth. An early suggestion for an eclipse of the B3V companion was made by Goranskij (2006) in December 2006 on the basis of $B$-band photometry showing the initial declining portion of the photometric profile (Fig. 1 open circles).
To have witnessed an eclipse in V838 Mon is indeed a lucky coincidence, in line with the continued surprises with which V838 Mon delights its fellow observers. Its duration was about 70 days from start to ingress to end of egress, and could have gone undetected if it had occurred during summer conjunction with the Sun. It is also a quite rare event. None have been detected in the previous 5 years of close photometric monitoring (Fig. 1). What caused this eclipse? There are two equally viable explanations.

The first one sees a dust cloud, absorbing according to the standard $R_{\mathrm{V}}=3.1$ law, that passes in front of the $\mathrm{B} 3 \mathrm{~V}$ star alone, without affecting the LtSG component. A direct integration of the resulting combined spectra energy distribution shows that a reddening by $E_{B-V}=0.55$ of the $\mathrm{B} 3 \mathrm{~V}$ would cause a drop in brightness of the combined binary system of $\Delta B \sim 1.15, \Delta V \sim 0.56$, and $\Delta R_{\mathrm{C}} \sim 0.07 \mathrm{mag}$, thus essentially identical to what observed. Such an eclipsing cloud should probably be gravitationally bound to the $\mathrm{B} 3 \mathrm{~V}+\mathrm{LtSG}$ binary. Its origin is unclear, perhaps connected to blobs of material thrown away by the LtSG at the time of heaviest mass loss during early outburst phases.

A second possibility is an eclipse of the B3V star by the LtSG companion. If the Pavlenko et al. (2007) estimate of a peak radius of $6000 R_{\odot}$ for the LtSG in winter 2002/03 is correct, the minimum orbital semi-major axis must be $6000 R_{\odot}(\equiv 28 \mathrm{AU})$ because at that time the $\mathrm{B} 3 \mathrm{~V}$ companion was still visible and therefore external to the LtSG outer radius. Adopting $7 M_{\odot}$ for the main sequence $\mathrm{B} 3 \mathrm{~V}$ companion, the orbital period corresponding to the $28 \mathrm{AU}$ lower limit to the orbital separation is $40 \mathrm{yr}$ for an LtSG mass of $7 M_{\odot}$, and $20 \mathrm{yr}$ for $40 M_{\odot}{ }^{1}$. The possibility of a heavy LtSG (progenitor) has been discussed by Munari et al. (2005) and Hirschi (2007). The eclipse lasted for $\leq 1 / 150$ of the orbital period, and in spite of the huge difference in radius of the two stars (B3V and LtSG) it did not go through a flat bottom. This means that the eclipse was a grazing one, by the outer layers of the LtSG atmosphere. A grazing eclipse in turn means that searching for past eclipses in photographic plate archive should not pay dividends. In fact, before the outburst, the radii of the two companion stars were similar according to photometry in quiescence (Goranskij et al. 2007). If we observe a grazing eclipse when the occulting body expanded by $\sim 100 \times$, it is quite probable that in quiescence the $\mathrm{B} 3 \mathrm{~V}$ passed well clear of the companion at orbital prospective conjunctions.

\section{Flaring of emission lines}

Shortly before the eclipse events discussed in the previous section, in late September 2006, the strength of [FeII] and FeII emission lines began increasing in V838 Mon, while $\mathrm{H} \alpha$ turned into emission for the first time since the initial phases of the 2002 outburst. The last time $\mathrm{H} \alpha$ was seen in emission was in April 2002 (cf. Munari et al. 2007). Since the strongest [FeII] and FeII emission lines cluster between 4200 and $4600 \AA$, their increase in intensity caused the brightening in the $B$-band visible in Fig. 1 just before the onset of the eclipse fading. This had no counterpart in the $V$-band due to the lower concentration of

1 Some authors preferred smaller maximum radii for the LtSG (e.g. Tylenda 2005). Two years after maximum expansion was reached, interferometric observations by Lane et al. (2005) gave an apparent radius of $0.92 \mathrm{mas}$, corresponding to a linear radius of $2000 R_{\odot}$ for a distance of $10 \mathrm{kpc}$. A $2000 R_{\odot}$ lower limit to the orbital separation would shorten the minimum orbital period to 7.5 and $4 \mathrm{yr}$ for LtSG masses of 7 and $40 M_{\odot}$, respectively. 


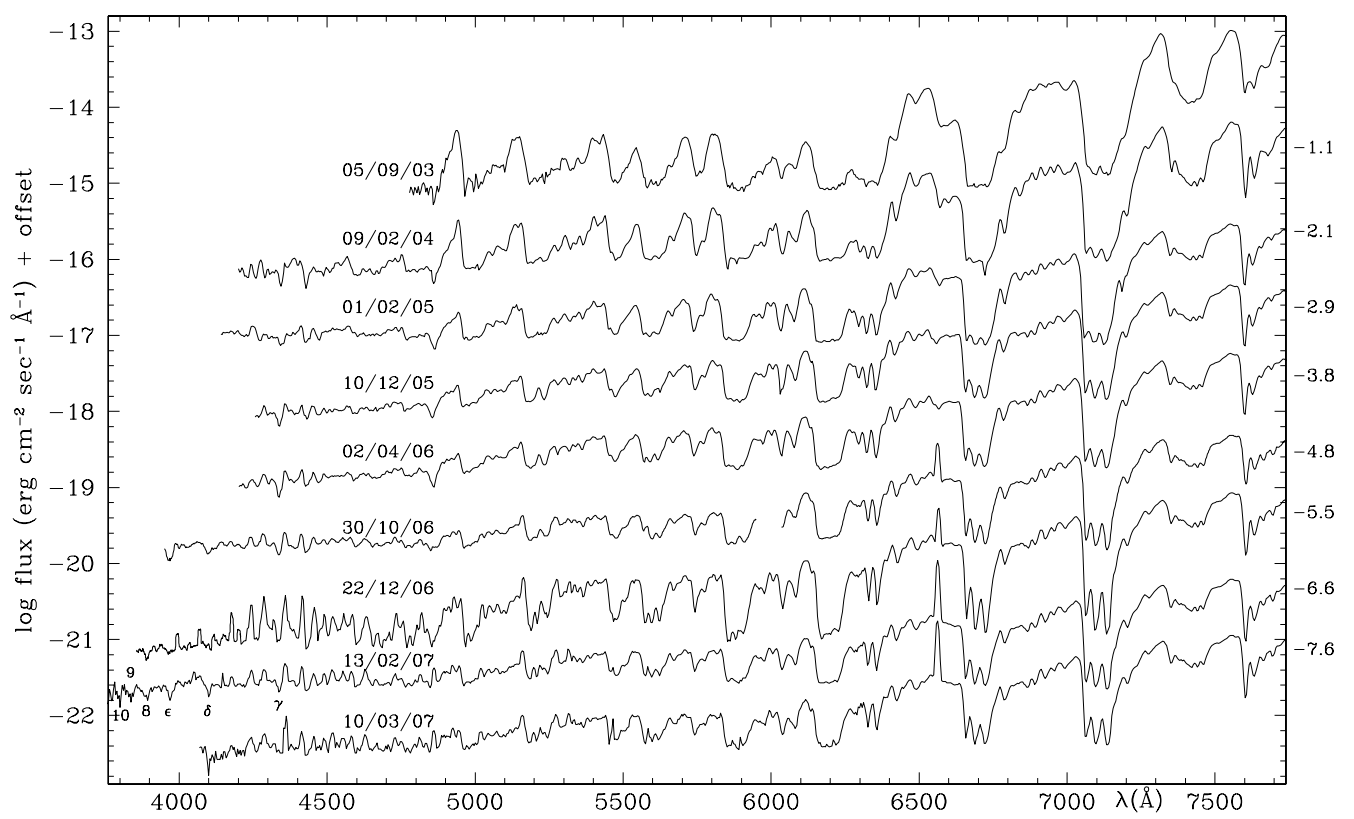

Fig. 2. Selection of Asiago $1.82 \mathrm{~m}$ + AFOSC spectra to highlight the general spectral evolution of V838 Mon during the last four years (cf. Munari et al. 2007). The spectra are on the same ordinate scale and are displaced for clarity by the log offsets listed to the right. The spectrum for Oct. 30, 2006 is from WHT (cf. Table 1), and has been degraded to the same resolution of the other Asiago spectra.

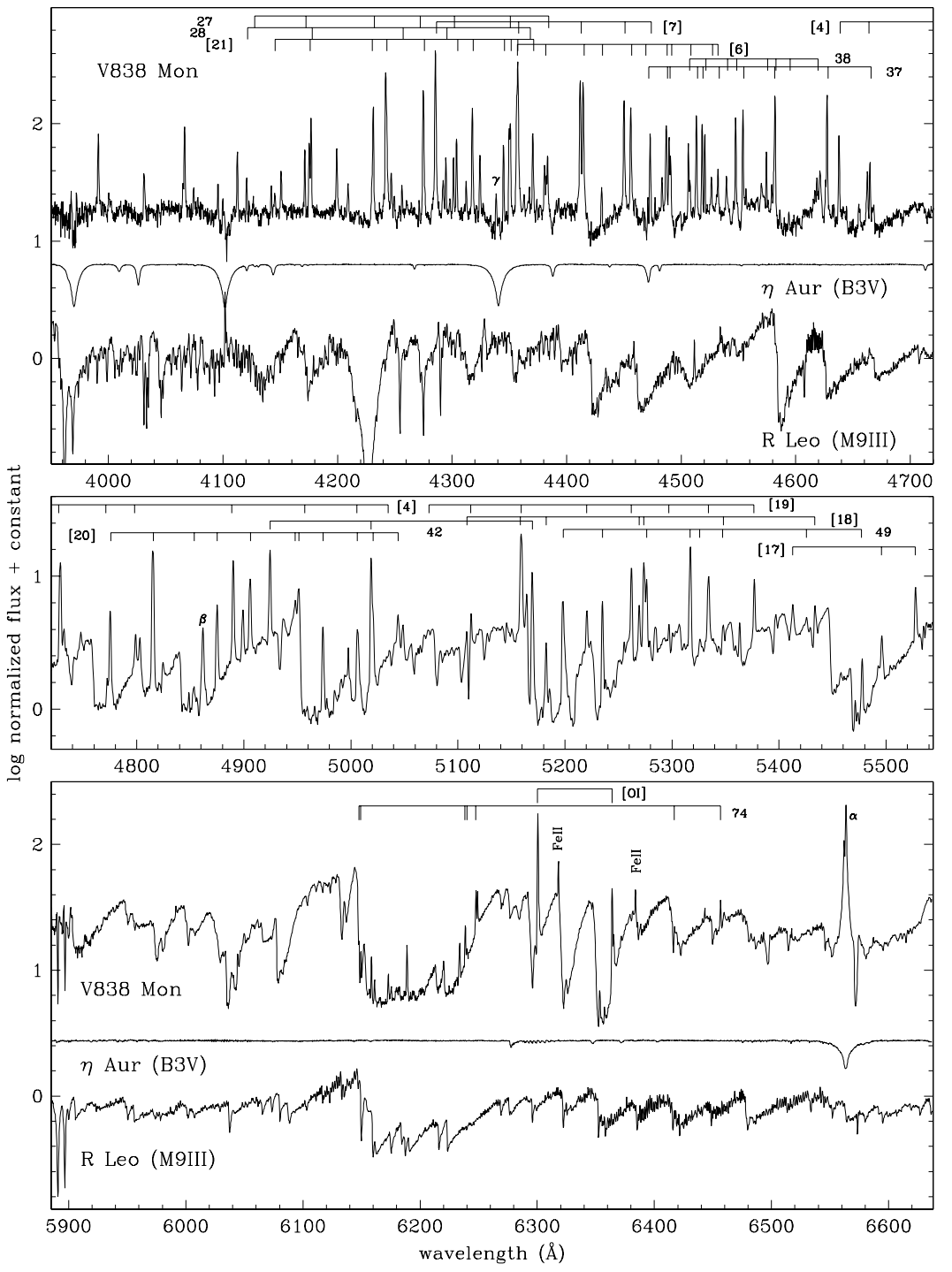

Fig. 3. The top and bottom panels show the WHT + ISIS spectrum of V838 Mon we obtained on December 26, 2006. The same night, and with an identical instrument set-up, we recorded for comparison the spectra of R Leo (M9III) and $\eta$ Aur (B3V). The central panel fills in the wavelength gap in the December 26, 2006 spectra by showing the corresponding portion of a lower resolution WHT + ISIS spectrum of V838 Mon for December 18, 2006. The spectra are continuum normalized and their ordinate step is the same in all panels. Note the logarithmic scale of ordinates required by the huge intensity of the emission lines. The emission lines belonging to strongest FeII and $[\mathrm{FeII}]$ multiplets are identified by their numbers (Table 4). 

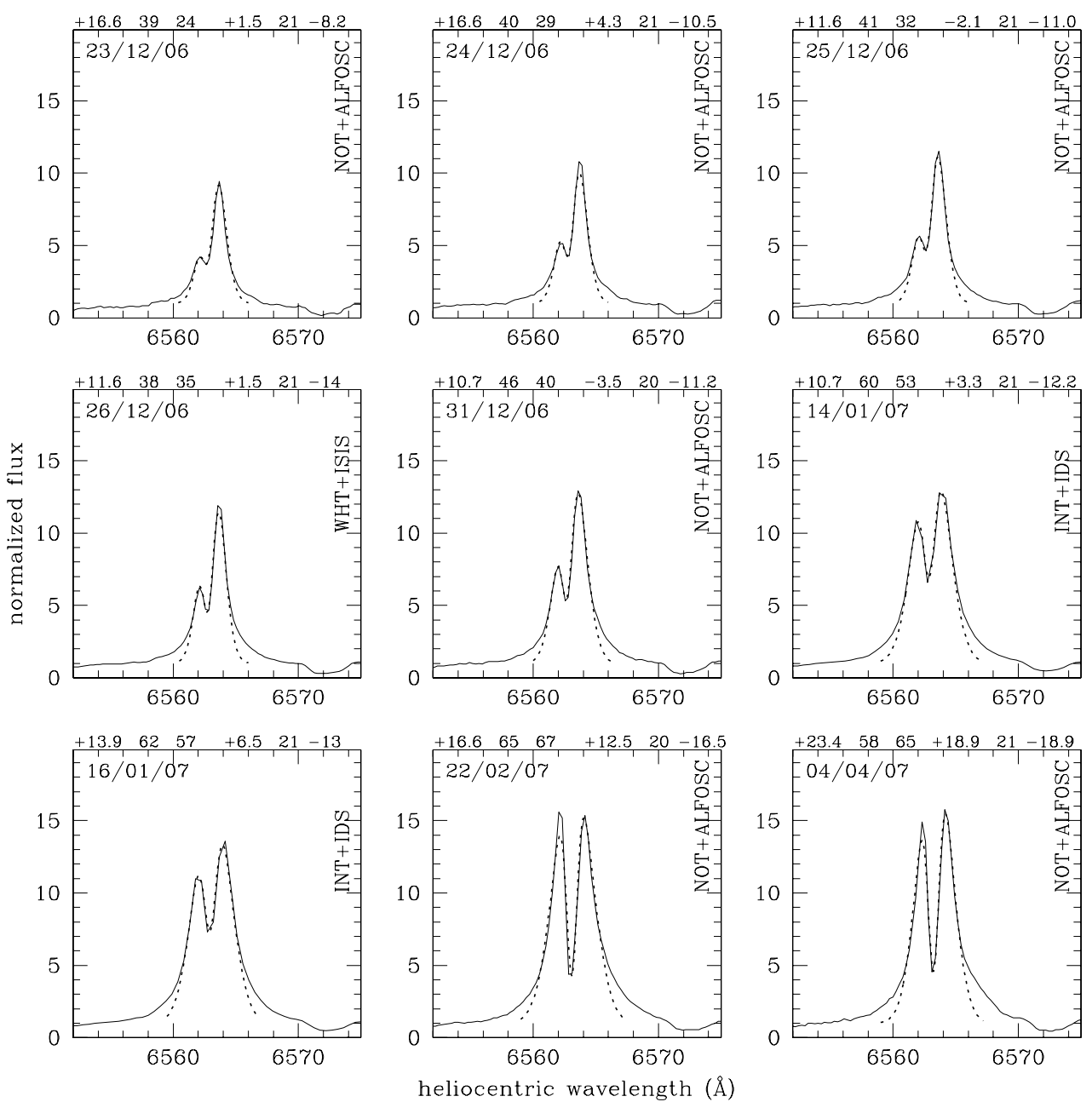

Fig. 4. Evolution of the $\mathrm{H} \alpha$ profile. Over-plotted is the fit (dotted line) with two Gaussians, one in emission and the other in absorption. The six numbers at the top of each panel represent the central heliocentric velocity (in $\mathrm{km} \mathrm{s}^{-1}$ ), the dispersion in velocity (in $\mathrm{km} \mathrm{s}^{-1}$ ) and the equivalent width $(\AA)$ of the emission and absorption fitting Gaussian, respectively. The acquisition in $\mathrm{ToO}$ mode for some of the spectra does not guarantee a strict control on the zero point of the wavelength scale.

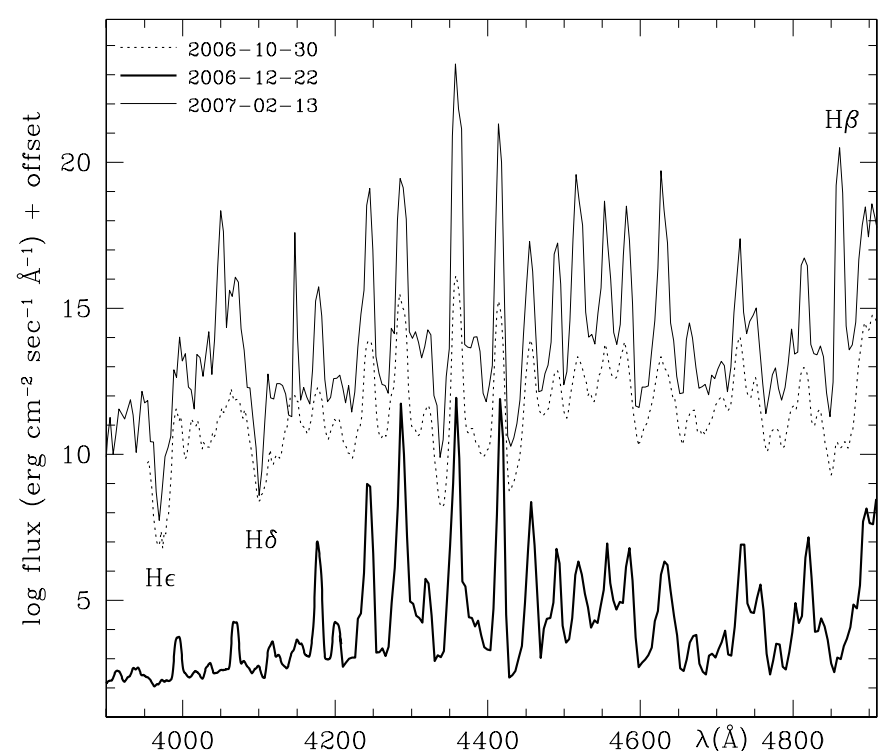

Fig. 5. Comparison of the blue portion of the V838 Mon spectra for October 30 and December 22, 2006 and February 13, 2007.

[FeII] and FeII emission lines and the much brighter background of the LtSG continuum, which steeply rises toward redder wavelengths. The fact that $(a)$ the emission lines began their growth in intensity before the eclipse event took place, $(b)$ the phenomenon smoothly continued after the eclipse-like phase was over; and (c) the $\mathrm{H} \alpha$ profile evolution was not in phase with the eclipse event, suggests that the two events (eclipse and emission line flaring) were not related and appeared at similar times just by chance.

On the high resolution, high $\mathrm{S} / \mathrm{N}$ WHT+ISIS spectra for December 18 and 26 (Fig. 3) we identified more than 200 emission lines. At that time, V838 Mon was going through the minimum of the eclipse, and the weaker continuum further increased the contrast of emission lines. The increase in the number of detected emission lines is $\geq 20 \times$ compared to published previous observations (e.g. Barsukova et al. 2007, observations covering 2004-2005). The radial velocity, integrated absolute flux and identification of the emission lines in our December 18 and 26 spectra, are given in Tables 3 and 4 (available electronic only). The vast majority of them belong to FeII (multiplets 20, 22, 27, 28, 32, 37, 38, 42, 43, 49, 54, 74) and [FeII] (multiplets $4,6,7,17,18,19,20,21,24,35,36)$, with forbidden lines much stronger than permitted ones. These lines are intrinsically sharp enough to be unresolved. The underlying continuum is highly structured because of the molecular absorption spectrum of the LtSG. This affects the accuracy of flux measurements, and in some cases can mimic the presence of P-Cyg absorptions. However, when the lines of the same multiplet are compared in velocity space, it is clear that FeII emission lines do not display absorption components.

Figure 4 displays the large evolution in intensity and shape of the $\mathrm{H} \alpha$ emission profile from December 23, 2006, to April 4,2007 . The profile is characterized by a sharp central reversal. We have fitted the $\mathrm{H} \alpha$ profiles with two Gaussians, a broader 
one in emission and a sharper one in absorption, and the results are given in Fig. 4. The sharp absorption component has kept a very stable velocity dispersion of $20.5 \mathrm{~km} \mathrm{~s}^{-1}$ and has increased its equivalent width by 2.3 times (from 8.2 to $18.9 \AA$ ), while the emission component has smoothly increased its velocity dispersion from 40 to $60 \mathrm{~km} \mathrm{~s}^{-1}$ and its equivalent width by 2.7 times (from 24 to $65 \AA$ ). The relative velocity of the emission component with respect to absorption has been declining from +13.5 to $+4.3 \mathrm{~km} \mathrm{~s}^{-1}$. Even if similarly double-peaked, the $\mathrm{H} \alpha$ profiles seem quite different from those originating from accretion disks, both optically thin and thick, of the type seen in interacting binaries (e.g. Horne \& Marsh 1986).

Detailed modeling of FeII, [FeII] emission line spectrum is a complex and not yet fully understood process (e.g. Viotti et al. 1999), far beyond the scope of this paper, and it will be discussed elsewhere together with modeling of the $\mathrm{H} \alpha$ profile evolution. However, it is intriguing to note that the velocity of the absorption component $\left(\left\langle R V_{\odot}^{\text {abs }}\right\rangle=+4.8 \mathrm{~km} \mathrm{~s}^{-1}\right.$ corresponding to $\left\langle R V_{\mathrm{LSR}}^{\mathrm{abs}}\right\rangle=-12.5$ in the Local Standard of Rest) is within $2 \mathrm{~km} \mathrm{~s}^{-1}$ of the $V_{\mathrm{LSR}}=-11 \mathrm{~km} \mathrm{~s}^{-1}$ of the CO radio emission detected in V838 Mon for the first time in December 2005 by Kaminski et al. (2007). The velocity of the $\mathrm{H} \alpha$ emission component $\left(\left\langle R V_{\odot}^{\mathrm{ems}}\right\rangle=+14.6 \mathrm{~km} \mathrm{~s}^{-1} \equiv\left\langle R V_{\mathrm{LSR}}^{\mathrm{ems}}\right\rangle=-2.8\right)$ is the same as that of FeII, [FeII] $\left(\left\langle R V_{\odot}\right\rangle=+15.3 \mathrm{~km} \mathrm{~s}^{-1} \equiv\right.$ $\left.\left\langle R V_{\mathrm{LSR}}\right\rangle=-2.1 \mathrm{~km} \mathrm{~s}^{-1}\right)$ and is not related to the other component seen in CO at $V_{\mathrm{LSR}}=+53.4 \mathrm{~km} \mathrm{~s}^{-1}$ by Kaminski et al. (2007) or in the $\mathrm{SiO}$ maser at $V_{\mathrm{LSR}}=+54.3 \mathrm{~km} \mathrm{~s}^{-1}$ by Deguchi et al. (2007).

Acknowledgements. We would like to thank the anonymous referee for his/her valuable comments and suggestions.

\section{References}

Barsukova, E. A., Goranskij, V. P., Abolmasov, P. K., Fabrika, S. N., et al. 2007, in The Nature of V838 Mon and Its Light Echo, ed. R. L. M. Corradi, \& U. Munari, ASP Conf. Ser., 363, 206

Bond, H., 2006, ATel, 966

Bond, H. 2007, in The Nature of V838 Mon and Its Light Echo, ed. R. L. M. Corradi, \& U. Munari, ASP Conf. Ser., 363, 130

Bond, H., Henden, A., Leavy, Z. G. et al. 2003, Nature, 422, 405

Boschi, F., \& Munari, U. 2004, A\&A, 418, 869
Brown, N. J. 2002, IAUC, 7785

Corradi, R. L. M., \& Munari, U. 2007, ed. The Nature of V838 Mon and Its Light Echo, ASP Conf. Ser., 363

Desidera, S., \& Munari, U. 2002, IAUC, 7982

Deguchi, S., Matsunaga, N., \& Fukushi, H. 2007, in The Nature of V838 Mon and Its Light Echo, ed. R. L. M. Corradi, \& U. Munari, ASP Conf. Ser., 363, 81

Goranskij, V. 2006, ATel, 964

Goranskij, V., Metlova, N. V., Shugarov, S. Yu., et al. 2007, in The Nature of V838 Mon and Its Light Echo, ed. R. L. M. Corradi, \& U. Munari, ASP Conf. Ser., 363, 214

Hajduk, M., Zijlstra, A. A., van Hoof, P. A. M., et al. 2007, MNRAS, 378, 1298 Henden, A. 2007, in The Nature of V838 Mon and Its Light Echo, ed. R. L. M. Corradi, \& U. Munari, ASP Conf. Ser., 363, 3

Henden, A., Munari, U., \& Schwartz, M. 2002, IAUC, 7859

Hirschi, R. 2007, in The Nature of V838 Mon and Its Light Echo, ed. R. L. M. Corradi, \& U. Munari, ASP Conf. Ser., 363, 257

Horne, K., \& Marsh, T. R. 1986, MNRAS, 218, 761

Iben, I. Jr., \& Tutukov, A. V. 1992, ApJ, 389, 369

Kaminski, T., et al. 2007, in The Nature of V838 Mon and Its Light Echo, ed. R. L. M. Corradi, \& U. Munari, ASP Conf. Ser., 363, 103

Kato, T., Miller, M., Szczerba, R., Tylenda, R. 2003, A\&A, 399, 695

Kimeswenger, S. 2007, in The Nature of V838 Mon and Its Light Echo, ed. R. L. M. Corradi, \& U. Munari, ASP Conf. Ser., 363, 197

Kulkarni, S. R., Ofek, E. O., Rau, A., et al. 2007, Nature, 447, 458

Lane, B. F., Retter, A., Thompson, R. R., Eisner, J. A., et al. 2005, ApJ, 622, L137

Lawlor, T. M. 2005, MNRAS, 361, 695

Lawlor, T. M. 2007, in The Nature of V838 Mon and Its Light Echo, ed. R. L. M. Corradi, \& U. Munari, ASP Conf. Ser., 363, 249

Martini, P., Wagner, R. M., Tomaney, A., et al. 1999, AJ, 118, 1034

Mayall, M. W. 1949, AJ, 54, R191

Mould, J., Cohen, J., Graham, J. R., et al. 1990, ApJ, 353, L35

Munari U., Henden A., Kiyota S., et al. 2002, A\&A, 389, L51

Munari, U., Henden, A., Vallenari, A., et al. 2005, A\&A 434, 1107

Munari, U., Navasardyan, H., Villanova, S., et al. 2007, in The Nature of V838 Mon and Its Light Echo, ed. R. L. M. Corradi, \& U. Munari, ASP Conf. Ser., 363, 13

Pavlenko, Ya. V., Kaminsky, B., Lyubchik, Y., Yakovina, L., et al. 2007, in The Nature of V838 Mon and Its Light Echo, ed. R. L. M. Corradi, \& U. Munari, ASP Conf. Ser., 363, 225

Retter, A., \& Marom, A. 2003, MNRAS, 345, L25

Retter, A., Zhang, B., Siess, L., \& Levinson, A. 2006, MNRAS, 370, 1573

Rich, R. M., Mould, J., Picard, A., et al. 1989, ApJ, 341, L51

Soaker, N., \& Tylenda, R. 2003, ApJ, 582, L105

Tylenda, R. 2005, A\&A, 436, 1009

Tylenda, R., \& Soker, N. 2006, A\&A, 451, 223

van Hoof, P. A. M., Bryce, M., Evans, A., et al. 2006, in Planetary Nebulae in our Galaxy and Beyond, ed. M. J. Barlow, \& R. H. Mendez, IAU Symp., 234,75

Viotti, R., Rossi, C., Baratta, G. B., et al. 1999, in ASP Conf. Ser., 179, 184 


\section{Online Material}


U. Munari et al.: V838 Mon and the events of fall 2006, Online Material p 2

Table 2. Our CCD photometry of V838 Mon since October 2006. HJD is the heliocentric JD - 2450000.

\begin{tabular}{|c|c|c|c|c|}
\hline HJD & $V$ & $B-V$ & $V-R_{\mathrm{C}}$ & $R_{\mathrm{C}}-I_{\mathrm{C}}$ \\
\hline 53704.8042 & $.347 \pm 0.007$ & $.122 \pm 0.029$ & $2.084 \pm 0.009$ & $2.799 \pm 0.0$ \\
\hline & $5.380 \pm 0.0$ & $117 \pm 0.031$ & $103 \pm 0.009$ & $.823 \pm 0.007$ \\
\hline 53842.6436 & $.296 \pm 0.0$ & $275 \pm 0.019$ & $103 \pm 0.009$ & $2.827 \pm 0.009$ \\
\hline 54007.9556 & $59 \pm 0$. & $.182 \pm 0.0$ & $2.171 \pm 0.017$ & $2.787 \pm 0.019$ \\
\hline 54009.9578 & $.252 \pm 0.0$ & $.176 \pm 0.021$ & $2.171 \pm 0.022$ & $2.776 \pm 0.022$ \\
\hline 54010. & & $1.234 \pm 0$. & $2.100 \pm 0$. & $2.785 \pm 0.038$ \\
\hline 54011.9523 & $5.239 \pm 0$. & $1.233 \pm 0.0$ & $2.161 \pm 0$. & $2.750 \pm 0.034$ \\
\hline 54012.9470 & $34 \pm 0$ & $1.234 \pm 0$ & $2.111 \pm 0$ & $2.806 \pm 0.034$ \\
\hline 54015.9420 & $.235 \pm 0$. & $1.202 \pm 0.028$ & $.137 \pm 0.015$ & $2.796 \pm 0.017$ \\
\hline 54016.9380 & $.218 \pm 0$ & $1.050 \pm 0.026$ & $2.127 \pm 0$. & $2.797 \pm 0.010$ \\
\hline 540 & & $134 \pm 0$. & $145 \pm 0$. & $2.795 \pm 0.015$ \\
\hline & $6 \pm 0$ & $895 \pm 0$ & $114 \pm 0$ & $.778 \pm 0$ \\
\hline 54062.8 & $2 \pm 0$ & $.336 \pm 0$ & $.409 \pm 0$ & $2.899 \pm 0.018$ \\
\hline 540 & $33 \pm c$ & $600 \pm 0$ & $.558 \pm 0$ & $2.909 \pm 0$ \\
\hline 540 & $6 \pm 0$ & $1.837 \pm 0$. & $595 \pm 0$ & $2.910 \pm 0.011$ \\
\hline 540 & $71 \pm 0$ & $1.756 \pm 0$. & $613 \pm 0$ & $2.898 \pm 0$. \\
\hline 540 & $71 \pm$ & $1.806 \pm 0$ & $.580 \pm 0$ & $2.905 \pm 0.013$ \\
\hline 540 & $64 \pm 0$ & $.804 \pm 0$ & $.551 \pm 0$ & $2.943 \pm 0$. \\
\hline 540 & $2 \pm$ & $1.753 \pm 0$ & $615 \pm 0$ & $2.888 \pm$ \\
\hline & $5 \pm$ & $511 \pm 0$ & $78 \pm 0$ & $00 \pm$ \\
\hline & $1 \pm$ & $618 \pm c$ & $15 \pm 0$ & $392 \pm$ \\
\hline & $4 \pm$ & $1.679 \pm 0$ & $525 \pm$ & $382 \pm$ \\
\hline 540 & $10 \pm$ & $1.709 \pm c$ & $.541 \pm 0$ & $2.892 \pm 0$ \\
\hline 540 & & $721 \pm 0$ & $564 \pm 0$ & $2.807 \pm$ \\
\hline & & $1.536 \pm 0$ & $413 \pm 0$ & $99 \pm c$ \\
\hline & $1 \pm$ & $1.444 \pm$ & $60 \pm 0$ & $784 \pm$ \\
\hline 541 & $2 \pm$ & $1.406 \pm 0$ & $2.391 \pm$ & $2.769 \pm 0$ \\
\hline 541 & $1 \pm$ & $311 \pm 0$ & $59 \pm 0$ & $2.658 \pm$ \\
\hline & & $196 \pm$ & $223 \pm$ & $700 \pm$ \\
\hline & $1 \pm$ & $307 \pm$ & $268 \pm$ & $730 \pm$ \\
\hline & $2 \pm$ & $221 \pm$ & $312 \pm 0$ & $2.748 \pm$ \\
\hline 541 & $14 \pm$ & $1.227 \pm 0$ & $183 \pm 0$ & $2.721 \pm 0.011$ \\
\hline & & $.247 \pm$ & $181 \pm 0$ & $700 \pm$ \\
\hline & $4 \pm$ & $231 \pm$ & $18 \pm$ & $70 \pm$ \\
\hline & $6 \pm$ & $211 \pm$ & $95 \pm$ & $23 \pm$ \\
\hline & $0 \pm$ & $1.201 \pm$ & $25 \pm$ & $622 \pm$ \\
\hline & $78 \pm$ & $1.221 \pm 0$ & $19 \pm 0$ & $2.703 \pm 0$ \\
\hline & & $61 \pm 0$ & $00 \pm 0$ & $79 \pm$ \\
\hline 54 & $9 \pm$ & $214 \pm 0$ & $06 \pm 0$ & $727 \pm$ \\
\hline & $0 \pm$ & $1.158 \pm 0$ & $2.137 \pm 0$ & $2.677 \pm 0$. \\
\hline 54126.6314 & $178 \pm$ & $1.240 \pm 0$ & $161 \pm 0$ & $2.637 \pm 0.013$ \\
\hline & & $1.210 \pm 0$ & $165 \pm 0$ & $.664 \pm$ \\
\hline & $1 \pm$ & $195 \pm$ & $29 \pm 0$ & $83 \pm$ \\
\hline & $1 \pm$ & $145 \pm 0$ & $82 \pm 0$ & $637 \pm 0$ \\
\hline & $103 \pm$ & $1.208 \pm 0$ & $2.082 \pm 0$ & $2.636 \pm 0$ \\
\hline & & $1.166 \pm 0$ & $2.101 \pm 0$ & $2.642 \pm 0$ \\
\hline & $8 \pm$ & $.208 \pm 0$ & $71 \pm 0$ & $65 \pm$ \\
\hline 54 & $5 \pm$ & $1.196 \pm 0$ & $29 \pm 0$ & $2.638 \pm$ \\
\hline 54145.6680 & $15.162 \pm 0$. & $1.223 \pm 0.027$ & $2.135 \pm 0.017$ & $2.674 \pm 0.012$ \\
\hline & $5.181 \pm 0$ & $1.257 \pm 0$. & $.170 \pm 0$. & $2.659 \pm 0.017$ \\
\hline & & $1.256 \pm 0$ & $156 \pm 0$ & $2.649 \pm$ \\
\hline 541 & $68 \pm 0$ & $1.244 \pm 0$ & $2.149 \pm 0$ & $2.645 \pm$ \\
\hline & $147 \pm 0$ & $1.250 \pm 0.0$ & $2.128 \pm 0.035$ & $2.651 \pm 0.022$ \\
\hline 541 & $5.176 \pm 0.0$ & $1.230 \pm 0.020$ & $2.158 \pm 0.016$ & $2.652 \pm 0.012$ \\
\hline & & $1.202 \pm 0$. & $2.089 \pm 0$. & $2.634 \pm 0.010$ \\
\hline & $5.198 \pm 0$ & $1.274 \pm 0$ & $2.181 \pm 0$ & $2.504 \pm 0.018$ \\
\hline 54160.6141 & $15.187 \pm 0.010$ & $1.203 \pm 0.027$ & $2.169 \pm 0.010$ & $2.661 \pm 0.006$ \\
\hline 54161.6427 & $15.181 \pm 0.009$ & $1.233 \pm 0.020$ & $2.147 \pm 0.011$ & $2.664 \pm 0.011$ \\
\hline 54166.615 & $5.156 \pm 0.0$ & $1.264 \pm 0.018$ & $2.153 \pm 0$. & $2.611 \pm 0.014$ \\
\hline & $87 \pm 0$ & $1.261 \pm 0$ & $2.146 \pm 0$ & $2.642 \pm 0$ \\
\hline 54175.6270 & $15.177 \pm 0.010$ & $1.224 \pm 0.026$ & $2.157 \pm 0.011$ & $2.664 \pm 0.011$ \\
\hline 54186.6278 & $15.268 \pm 0.020$ & $1.360 \pm 0.037$ & $2.270 \pm 0.024$ & $2.688 \pm 0.026$ \\
\hline 54195.6474 & $15.136 \pm 0.011$ & $1.345 \pm 0.032$ & $2.130 \pm 0.017$ & $2.631 \pm 0.026$ \\
\hline
\end{tabular}




\section{U. Munari et al.: V838 Mon and the events of fall 2006, Online Material $p 3$}

Table 3. Identification of emission lines in the WHT + ISIS spectra of V838 Mon for December 18 ( $4724 \leq \lambda \leq 5544 \AA$ ) and December 26 $\left(\lambda \leq 4723\right.$ and $\lambda \geq 5862 \AA$ ). The integrated line fluxes are given both in units of erg $\mathrm{cm}^{-2} \mathrm{~s}^{-1}$ as well as relative to $\mathrm{H} \beta$. The wavelengths are heliocentric and the fluxes are not corrected for reddening.

\begin{tabular}{|c|c|c|c|c|c|c|c|c|c|c|c|c|c|c|}
\hline$\lambda_{\odot}^{o b s}(\AA)$ & Flux & $\frac{\mathrm{F}_{\lambda}}{\mathrm{F}_{H \beta}}$ & $\begin{array}{c}\text { E.W. } \\
(\AA)\end{array}$ & ident & $\lambda_{\odot}^{o b s}(\AA)$ & Flux & $\frac{\mathrm{F}_{\lambda}}{\mathrm{F}_{H \beta}}$ & $\begin{array}{c}\text { E.W. } \\
(\AA)\end{array}$ & ident & $\lambda_{\odot}^{o b s}(\AA)$ & Flux & $\frac{\mathrm{F}_{\lambda}}{\mathrm{F}_{H \beta}}$ & $\begin{array}{c}\text { E.W. } \\
(\AA)\end{array}$ & ident \\
\hline 3914.168 & $1.955 \mathrm{E}-16$ & 0.091 & 0.881 & & 4369.255 & $1.468 \mathrm{e}-16$ & 0.069 & 0.414 & FeII 28 & 4950.899 & $1.166 \mathrm{e}-15$ & 0.546 & 0.761 & [FeII] 20 \\
\hline 3915.746 & $9.701 \mathrm{E}-17$ & 0.045 & 0.430 & & 4372.325 & $1.289 \mathrm{e}-15$ & 0.603 & 3.484 & [FeII] 21 & 4957.567 & $9.136 \mathrm{e}-17$ & 0.043 & 0.277 & [FeII] 4 \\
\hline 3938.876 & $2.420 \mathrm{E}-16$ & 0.113 & 0.999 & & 4382.591 & $5.544 \mathrm{e}-16$ & 0.259 & 1.550 & {$[\mathrm{FeII}] 6$} & 4973.627 & $1.947 \mathrm{e}-15$ & 0.911 & 6.099 & [FeII $] 20$ \\
\hline 3945.643 & $1.670 \mathrm{E}-16$ & 0.078 & 0.762 & & 4384.093 & $3.027 \mathrm{e}-16$ & 0.142 & 0.850 & [FeII $] 36$ & 4997.287 & $6.301 \mathrm{e}-16$ & 0.295 & 1.071 & \\
\hline 3969.901 & $2.490 \mathrm{E}-16$ & 0.117 & 1.514 & $\mathrm{H} \epsilon$ & 4385.217 & $6.392 \mathrm{e}-16$ & 0.299 & 1.802 & FeII 27 & 5005.792 & $2.887 \mathrm{e}-16$ & 0.135 & 0.688 & [FeII] 20 \\
\hline 3974.662 & $2.476 \mathrm{E}-16$ & 0.116 & 1.407 & & 4399.782 & $1.433 \mathrm{e}-16$ & 0.067 & 0.259 & FeII 20 & 5007.007 & $6.896 \mathrm{e}-16$ & 0.323 & 1.591 & {$[\mathrm{Fe} \mathrm{II}] 4$} \\
\hline 3993.008 & $9.988 \mathrm{e}-16$ & 0.467 & 3.779 & [Ni II $] 4$ & 4402.687 & $4.885 \mathrm{e}-17$ & 0.023 & 0.131 & [FeII $] 36$ & 5018.640 & $6.799 \mathrm{e}-15$ & 3.182 & 13.70 & FeII 42 \\
\hline 4017.244 & $7.673 \mathrm{e}-17$ & 0.036 & 0.312 & {$[\mathrm{Fe}$ II $] 24$} & 4413.662 & $4.618 \mathrm{e}-15$ & 2.161 & 13.68 & {$[\mathrm{FeII}] 7$} & 5020.470 & $1.976 \mathrm{e}-15$ & 0.925 & 3.967 & [FeII $] 20$ \\
\hline 4033.719 & $1.021 \mathrm{e}-16$ & 0.048 & 0.456 & {$[\mathrm{Fe}$ II $] 24$} & 4432.343 & $3.592 \mathrm{e}-16$ & 0.168 & 1.413 & {$[\mathrm{FeII}] 6$} & 5043.886 & $1.399 \mathrm{e}-15$ & 0.655 & 1.366 & [FeII $] 20$ \\
\hline 4066.897 & $1.856 \mathrm{e}-16$ & 0.087 & 0.669 & NiII 11 & 4439.661 & $5.947 \mathrm{e}-17$ & 0.028 & 0.219 & [FeII] 36 & 5048.337 & $1.098 \mathrm{e}-15$ & 0.514 & 1.213 & {$[\mathrm{FeII}]$} \\
\hline 4068.350 & $1.165 \mathrm{e}-15$ & 0.545 & 4.199 & [SII] 1 & 4451.988 & $3.096 \mathrm{e}-15$ & 1.449 & 7.827 & {$[\mathrm{FeII}] 7$} & 5071.859 & $4.012 \mathrm{e}-16$ & 0.188 & 0.255 & {$[\mathrm{Fe}$ II $] 19$} \\
\hline 4076.368 & $1.936 \mathrm{e}-16$ & 0.091 & 0.730 & {$[\mathrm{SII}] 1$} & 4457.859 & $2.658 \mathrm{e}-15$ & 1.244 & 7.050 & [FeII] 6 & 5083.447 & $5.097 \mathrm{e}-16$ & 0.239 & 0.5085 & {$[\mathrm{Fe}$ II $] 35$} \\
\hline 4079.870 & $8.076 \mathrm{e}-17$ & 0.038 & 0.286 & [Fe II $] 24$ & 4461.023 & $5.772 \mathrm{e}-17$ & 0.027 & 0.169 & [Ni II $] 10$ & 5107.697 & $9.084 \mathrm{e}-16$ & 0.425 & 0.733 & {$\left[\begin{array}{lll}\mathrm{Fe} & \mathrm{II}\end{array}\right]$} \\
\hline 4084.290 & $4.009 \mathrm{e}-17$ & 0.019 & 0.166 & [Fe II $] 24$ & 4470.143 & $1.157 \mathrm{e}-16$ & 0.054 & 0.482 & {$[\mathrm{FeII}] 6$} & 5112.083 & $1.244 \mathrm{e}-15$ & 0.582 & 0.820 & {$\left[\begin{array}{lll}\mathrm{Fe} & \mathrm{II}\end{array}\right] 19$} \\
\hline 4097.349 & $2.196 \mathrm{e}-16$ & 0.103 & 0.881 & & 4472.726 & $1.605 \mathrm{e}-16$ & 0.075 & 0.595 & FeII 37 & 5158.792 & $1.348 \mathrm{e}-14$ & 6.308 & 8.426 & $e$ \\
\hline 4101.669 & $1.274 \mathrm{e}-16$ & 0.060 & 0.610 & $\mathrm{H} \delta$ & 4474.787 & $1.355 \mathrm{e}-15$ & 0.634 & 4.585 & {$[\mathrm{FeII}] 7$} & 5163.993 & $3.669 \mathrm{e}-15$ & 1.717 & 3.814 & [FeII] 35 \\
\hline 4102.885 & $9.526 \mathrm{e}-17$ & 0.045 & 0.471 & & 4478.958 & $1.305 \mathrm{e}-16$ & 0.061 & 0.432 & {$[\mathrm{Fe}$ II $]$} & 5169.236 & $7.032 \mathrm{e}-15$ & 3.291 & 14.08 & FeII 42 \\
\hline 4114.397 & $5.982 \mathrm{e}-16$ & 0.280 & 2.093 & [FeII] 23 & 4485.133 & $3.404 \mathrm{e}-16$ & 0.159 & 0.907 & {$[\mathrm{NiII}] 3$} & 5182.010 & $1.690 \mathrm{e}-15$ & 0.791 & 3.849 & [FeII] 18 \\
\hline 4122.504 & $2.503 \mathrm{e}-16$ & 0.117 & 0.913 & FeII 28 & 4488.785 & $1.670 \mathrm{e}-15$ & 0.781 & 4.063 & $d$ & 5197.798 & $4.702 \mathrm{e}-15$ & 2.200 & 7.769 & FeII 49 \\
\hline 4128.286 & $9.345 \mathrm{e}-17$ & 0.044 & 0.328 & FeII 27 & 4492.508 & $7.620 \mathrm{e}-16$ & 0.357 & 2.078 & [FeII] 6 & 5220.175 & $2.063 \mathrm{e}-15$ & 0.965 & 1.722 & {$[\mathrm{FeII}] 19$} \\
\hline 4144.038 & $2.010 \mathrm{e}-16$ & 0.094 & 0.718 & FeII 45 & 4508.092 & $8.930 \mathrm{e}-16$ & 0.418 & 2.684 & FeII 38 & 5234.710 & $3.698 \mathrm{e}-15$ & 1.730 & 6.277 & FeII 49 \\
\hline 4145.810 & $4.687 \mathrm{e}-17$ & 0.022 & 0.169 & [Fe II $] 21$ & 4509.476 & $3.799 \mathrm{e}-16$ & 0.178 & 1.132 & [FeII] 6 & 5261.764 & $6.929 \mathrm{e}-15$ & 3.242 & 5.186 & [FeII] 19 \\
\hline 4147.130 & $1.946 \mathrm{e}-16$ & 0.091 & 0.700 & {$\left[\begin{array}{lll}\mathrm{Ni} & \mathrm{II}\end{array}\right]$} & 4515.090 & $2.044 \mathrm{e}-15$ & 0.956 & 5.751 & FeII 37 & 5268.990 & $2.890 \mathrm{e}-15$ & 1.352 & 2.358 & {$[\mathrm{FeII}] 18$} \\
\hline 4152.612 & $3.787 \mathrm{e}-16$ & 0.177 & 1.178 & & 4520.070 & $1.440 \mathrm{e}-15$ & 0.674 & 4.343 & FeII 37 & 5273.560 & $8.646 \mathrm{e}-15$ & 4.046 & 6.392 & [FeII] 18 \\
\hline 4173.296 & $6.579 \mathrm{e}-16$ & 0.308 & 2.285 & FeII 27 & 4522.458 & $1.113 \mathrm{e}-15$ & 0.521 & 3.225 & FeII 38 & 5276.062 & $4.482 \mathrm{e}-15$ & 2.097 & 3.344 & FeII 49 \\
\hline 4177.177 & $1.015 \mathrm{e}-15$ & 0.475 & 3.562 & [FeII] 21 & 4528.277 & $3.580 \mathrm{e}-16$ & 0.168 & 0.949 & [FeII] 6 & 5284.133 & $2.233 \mathrm{e}-15$ & 1.045 & 1.907 & FeII 41 \\
\hline 4178.730 & $1.591 \mathrm{e}-15$ & 0.745 & 5.632 & FeII 28 & 4532.806 & $1.602 \mathrm{e}-16$ & 0.075 & 0.423 & [FeII] 6 & 5296.950 & $9.714 \mathrm{e}-16$ & 0.455 & 0.697 & [FeII] 19 \\
\hline 4201.154 & $8.327 \mathrm{e}-16$ & 0.390 & 2.466 & {$\left[\begin{array}{lll}\mathrm{Ni} & \mathrm{II}\end{array}\right]$} & 4533.953 & $3.511 \mathrm{e}-16$ & 0.164 & 0.930 & FeII 37 & 5316.708 & $1.175 \mathrm{e}-14$ & 5.498 & 8.765 & FeII 49 \\
\hline 4205.278 & $3.713 \mathrm{e}-17$ & 0.017 & 0.131 & FeII 22 & 4541.415 & $3.805 \mathrm{e}-16$ & 0.178 & 1.022 & FeII 38 & 5325.739 & $6.505 \mathrm{e}-16$ & 0.304 & 0.380 & FeII 49 \\
\hline 4211.006 & $2.795 \mathrm{e}-16$ & 0.131 & 0.901 & {$[\mathrm{FeII}]$} & 4547.451 & $1.873 \mathrm{e}-16$ & 0.088 & 0.665 & & 5333.787 & $6.369 \mathrm{e}-15$ & 2.980 & 3.845 & [FeII] 19 \\
\hline 4231.896 & $5.303 \mathrm{e}-16$ & 0.248 & 1.915 & [FeII] 21 & 4549.318 & $1.906 \mathrm{e}-15$ & 0.892 & 6.735 & FeII 38 & 5347.367 & $1.383 \mathrm{e}-15$ & 0.647 & 0.969 & $f$ \\
\hline 4233.065 & $2.458 \mathrm{e}-15$ & 1.150 & 8.106 & FeII 27 & 4555.725 & $2.036 \mathrm{e}-15$ & 0.953 & 5.172 & FeII 37 & 5362.948 & $1.845 \mathrm{e}-15$ & 0.863 & 1.511 & FeII 48 \\
\hline 4244.033 & $6.187 \mathrm{e}-15$ & 2.895 & 17.97 & [FeII] 21 & 4558.462 & $1.205 \mathrm{e}-16$ & 0.056 & 0.296 & FeII 20 & 5376.509 & $5.346 \mathrm{e}-15$ & 2.502 & 2.909 & [FeII] 19 \\
\hline 4248.812 & $4.465 \mathrm{e}-16$ & 0.209 & 1.354 & {$[\mathrm{Ni}$ II $] 4$} & 4571.931 & $1.938 \mathrm{e}-16$ & 0.091 & 0.408 & & 5412.494 & $3.882 \mathrm{e}-15$ & 1.817 & 2.077 & {$[\mathrm{FeII}] 17$} \\
\hline 4251.324 & $1.966 \mathrm{e}-16$ & 0.092 & 0.676 & {$[\mathrm{FeII}]$} & 4576.210 & $6.520 \mathrm{e}-16$ & 0.305 & 1.649 & FeII 38 & 5425.251 & $6.248 \mathrm{e}-16$ & 0.292 & 0.204 & FeII 49 \\
\hline 4258.069 & $2.512 \mathrm{e}-16$ & 0.118 & 0.806 & FeII 28 & 4577.707 & $3.843 \mathrm{e}-17$ & 0.018 & 0.101 & FeII 54 & 5433.327 & $2.930 \mathrm{e}-15$ & 1.371 & 1.584 & [FeII] 18 \\
\hline 4266.264 & $5.328 \mathrm{e}-17$ & 0.025 & 0.161 & [FeII] 36 & 4582.638 & $4.620 \mathrm{e}-16$ & 0.216 & 1.374 & FeII 37 & 5477.219 & $1.572 \mathrm{e}-15$ & 0.736 & 2.763 & FeII 49 \\
\hline 4273.142 & $1.079 \mathrm{e}-16$ & 0.050 & 0.388 & FeII 27 & 4583.669 & $2.811 \mathrm{e}-15$ & 1.315 & 8.397 & FeII 38 & 5495.648 & $2.386 \mathrm{e}-15$ & 1.117 & 2.337 & [FeII] 17 \\
\hline 4276.742 & $3.525 \mathrm{e}-15$ & 1.650 & 11.97 & [FeII] 21 & 4587.988 & $7.871 \mathrm{e}-17$ & 0.037 & 0.280 & & 5527.233 & $5.134 \mathrm{e}-15$ & 2.402 & 2.532 & {$[\mathrm{FeII}] 17$} \\
\hline 4277.790 & $3.012 \mathrm{e}-16$ & 0.141 & 0.942 & FeII 32 & 4595.896 & $8.713 \mathrm{e}-17$ & 0.041 & 0.327 & FeII 38 & 5869.959 & $6.091 \mathrm{e}-16$ & 0.285 & 1.268 & {$[\mathrm{Fe} \mathrm{II}]$} \\
\hline 4285.018 & $8.620 \mathrm{e}-18$ & 0.004 & 0.021 & {$[\mathrm{Ni} \mathrm{II}] 4$} & 4620.318 & $2.259 \mathrm{e}-16$ & 0.106 & 0.506 & FeII 38 & 5884.880 & $1.536 \mathrm{e}-16$ & 0.072 & 0.369 & $\mathrm{Fe}$ II \\
\hline 4296.392 & $5.811 \mathrm{e}-16$ & 0.272 & 1.792 & FeII 28 & 4639.562 & $1.311 \mathrm{e}-15$ & 0.613 & 3.893 & [FeII] 4 & 6044.056 & $4.384 \mathrm{e}-16$ & 0.205 & 0.468 & {$[\mathrm{Fe} \mathrm{II}]$} \\
\hline 4299.944 & $1.186 \mathrm{e}-16$ & 0.055 & 0.362 & TiII 41 & 4656.823 & $1.241 \mathrm{e}-16$ & 0.058 & 0.445 & FeII 43 & 6147.661 & $2.002 \mathrm{e}-16$ & 0.094 & 0.237 & FeII 74 \\
\hline 4303.001 & $5.733 \mathrm{e}-16$ & 0.268 & 1.701 & FeII 27 & 4657.796 & $9.865 \mathrm{e}-17$ & 0.046 & 0.350 & & 6149.160 & $2.995 \mathrm{e}-16$ & 0.140 & 0.505 & FeII 74 \\
\hline 4305.789 & $1.073 \mathrm{e}-15$ & 0.502 & 3.179 & [FeII] 21 & 4664.248 & $5.509 \mathrm{e}-16$ & 0.258 & 1.680 & $a$ & 6158.064 & $4.301 \mathrm{e}-16$ & 0.201 & 1.073 & $\mathrm{Fe}$ II \\
\hline 4307.862 & $7.637 \mathrm{e}-17$ & 0.036 & 0.276 & TiII 41 & 4666.613 & $6.000 \mathrm{e}-16$ & 0.281 & 1.817 & FeII 37 & 6160.959 & $1.671 \mathrm{e}-16$ & 0.078 & 0.441 & Fe II \\
\hline 4310.063 & $3.499 \mathrm{e}-17$ & 0.016 & 0.118 & {$[\mathrm{Ni}$ II $] 10$} & 4670.032 & $1.298 \mathrm{e}-16$ & 0.061 & 0.454 & & 6172.577 & $1.978 \mathrm{e}-16$ & 0.093 & 0.524 & Fe II \\
\hline 4312.643 & $3.944 \mathrm{e}-17$ & 0.018 & 0.114 & TiII 41 & 4572.389 & $1.068 \mathrm{E}-16$ & 0.050 & 0.100 & [Ni II $] 10$ & 6233.435 & $4.697 \mathrm{e}-16$ & 0.220 & 0.822 & FeII \\
\hline 4314.131 & $2.570 \mathrm{e}-16$ & 0.120 & 0.759 & FeII 32 & 4728.271 & $3.873 \mathrm{e}-15$ & 1.812 & 7.105 & {$[\mathrm{FeII}] 4$} & 6238.295 & $5.953 \mathrm{e}-16$ & 0.279 & 0.773 & FeII 74 \\
\hline 4314.797 & $2.734 \mathrm{e}-17$ & 0.013 & 0.082 & TiII 41 & 4731.573 & $4.278 \mathrm{e}-16$ & 0.200 & 0.717 & FeII 43 & 6239.742 & $1.569 \mathrm{e}-16$ & 0.073 & 0.186 & FeII 74 \\
\hline 4319.530 & $2.422 \mathrm{e}-15$ & 1.133 & 7.153 & {$[\mathrm{FeII}] 21$} & 4772.124 & $2.987 \mathrm{e}-16$ & 0.140 & 0.993 & {$[\mathrm{FeII}] 4$} & 6247.441 & $1.278 \mathrm{e}-15$ & 0.598 & 0.887 & FeII 74 \\
\hline 4326.189 & $7.842 \mathrm{e}-16$ & 0.367 & 2.348 & {$[\mathrm{Ni}$ II $] 3$} & 4774.911 & $1.889 \mathrm{e}-15$ & 0.884 & 6.730 & {$[\mathrm{FeII}] 20$} & 6248.792 & $1.170 \mathrm{e}-15$ & 0.547 & 0.741 & $\mathrm{Fe}$ II \\
\hline 4326.990 & $3.656 \mathrm{e}-17$ & 0.017 & 0.111 & FeII 20 & 4798.504 & $6.191 \mathrm{e}-16$ & 0.290 & 1.152 & $b$ & 6300.224 & $1.445 \mathrm{e}-14$ & 6.762 & 10.16 & [OI] 1 \\
\hline 4328.883 & $5.194 \mathrm{e}-17$ & 0.024 & 0.164 & & 4802.822 & $7.431 \mathrm{e}-16$ & 0.348 & 1.597 & & 6317.851 & $2.519 \mathrm{e}-15$ & 1.179 & 0.580 & Fe II \\
\hline 4329.350 & $3.365 \mathrm{e}-17$ & 0.016 & 0.106 & 36 & 4814.759 & $6.370 \mathrm{e}-15$ & 2.981 & 16.61 & [FeII $] 20$ & 6363.668 & $4.714 \mathrm{e}-15$ & 2.206 & 4.166 & [OI] 1 \\
\hline 4338.403 & $4.900 \mathrm{e}-17$ & 0.023 & 0.232 & FeII 32 & 4853.046 & $1.968 \mathrm{e}-16$ & 0.092 & 0.565 & {$[\mathrm{FeII}] 20$} & 6383.585 & $1.970 \mathrm{e}-15$ & 0.922 & 0.399 & $\mathrm{Fe} I \mathrm{II}$ \\
\hline 4340.426 & $2.996 \mathrm{e}-16$ & 0.140 & 1.210 & $\mathrm{H} \gamma$ & 4861.522 & $2.137 \mathrm{e}-15$ & 1.000 & 7.865 & $\mathrm{H} \beta$ & 6416.845 & $5.071 \mathrm{e}-16$ & 0.237 & 0.119 & FeII 74 \\
\hline 4346.732 & $1.025 \mathrm{e}-15$ & 0.480 & 4.051 & [FeII] 21 & 4874.756 & $2.205 \mathrm{e}-15$ & 1.032 & 5.183 & [FeII] 20 & 6456.200 & $3.936 \mathrm{e}-15$ & 1.842 & 0.633 & FeII 74 \\
\hline 4351.815 & $1.322 \mathrm{e}-15$ & 0.619 & 4.524 & FeII 27 & 4889.837 & $5.372 \mathrm{e}-15$ & 2.514 & 8.231 & [FeII 4 & 6491.115 & $7.263 \mathrm{e}-16$ & 0.340 & 0.119 & $\mathrm{Fe}$ II \\
\hline 4352.670 & $1.646 \mathrm{e}-15$ & 0.770 & 5.576 & [FeII] 21 & 4898.803 & $1.494 \mathrm{e}-15$ & 0.699 & 2.119 & {$\left[\begin{array}{ll}\mathrm{Fe} & \mathrm{II}\end{array}\right]$} & 6492.932 & $9.711 \mathrm{e}-16$ & 0.454 & 0.155 & Fe II \\
\hline 4358.166 & $2.363 \mathrm{e}-15$ & 1.106 & 6.741 & $c$ & 4905.579 & $3.385 \mathrm{e}-15$ & 1.584 & 4.127 & {$[\mathrm{FeII}] 20$} & 6516.938 & $9.349 \mathrm{e}-16$ & 0.437 & 0.123 & Fe II \\
\hline 4359.213 & $6.515 \mathrm{e}-15$ & 3.049 & 18.58 & {$[\mathrm{FeII}] 7$} & 4924.170 & $6.343 \mathrm{e}-15$ & 2.968 & 6.413 & FeII 42 & 6562.940 & $2.234 \mathrm{e}-13$ & 104.5 & 24.69 & $\mathrm{H} \alpha$ \\
\hline 4364.722 & $1.406 \mathrm{e}-16$ & 0.066 & 0.389 & & 4947.622 & $8.234 \mathrm{e}-16$ & 0.385 & 0.562 & [FeII $] 20$ & 6586.655 & $8.059 \mathrm{e}-16$ & 0.377 & 0.104 & Fe II \\
\hline
\end{tabular}

$a$ : blend of [FeII] 44664.440 and $[\mathrm{FeII}] 44665.645 \AA$

$b$ : blend of [FeII] 44798.274 and [FeII] $44799.293 \AA$

$c$ : blend of [FeII] 64358.095 and [Fe II] $214358.471 \AA$

$d$ : blend of [FeII] 64488.749 and Fe II $374489.183 \AA$

$e$ : blend of [FeII] 185347.653 and Fe II $495347.228 \AA$ 
Table 4. The emission lines in Table 3 are arranged in multiplets and their intensity - this time corrected for $E_{B-V}=0.87$ reddening (Munari et al. 2005 ) - is given relative to the strongest line of each multiplet. The wavelengths are corresponding laboratory ones.

\begin{tabular}{|c|c|c|c|c|c|c|c|c|c|c|c|}
\hline $\begin{array}{l}\lambda_{\text {lab }} \\
(\AA)\end{array}$ & $\begin{array}{c}\text { dered } \\
\mathrm{F}_{\lambda} / \mathrm{F}_{\lambda}^{P}\end{array}$ & $\begin{array}{c}\text { E.W. } \\
(\AA)\end{array}$ & multiplet & $\begin{array}{c}\lambda_{\text {lab }} \\
(\AA)\end{array}$ & $\begin{array}{c}\text { dered. } \\
\mathrm{F}_{\lambda} / \mathrm{F}_{\lambda}^{P}\end{array}$ & $\begin{array}{c}\text { E.W. } \\
(\AA)\end{array}$ & multiplet & $\begin{array}{l}\lambda_{\text {lab }} \\
(\AA)\end{array}$ & $\begin{array}{c}\text { dered. } \\
\mathrm{F}_{\lambda} / \mathrm{F}_{\lambda}^{P}\end{array}$ & $\begin{array}{c}\text { E.W. } \\
(\AA)\end{array}$ & multiplet \\
\hline 4639.666 & 0.342 & 3.893 & [FeII] 4 & 4146.649 & 0.008 & 0.169 & [FeII] 21 & 4508.283 & 0.318 & 2.684 & FeII 38 \\
\hline 4665.043 & 0.144 & 1.680 & {$[\mathrm{FeII}] 4^{a}$} & 4177.196 & 0.164 & 3.562 & [FeII] 21 & 4522.634 & 0.396 & 3.225 & FeII 38 \\
\hline 4728.068 & 0.721 & 7.105 & [FeII] 4 & 4231.554 & 0.086 & 1.915 & [FeII] 21 & 4541.523 & 0.135 & 1.022 & FeII 38 \\
\hline 4772.062 & 0.035 & 0.993 & [FeII] 4 & 4244.143 & 1.000 & 17.97 & [FeII] 21 & 4549.467 & 0.678 & 6.735 & FeII 38 \\
\hline 4798.783 & 0.115 & 1.152 & {$[\mathrm{FeII}] 4^{b}$} & 4276.829 & 0.570 & 11.97 & [FeII] 21 & 4576.331 & 0.232 & 1.649 & FeII 38 \\
\hline 4889.617 & 1.000 & 8.231 & [FeII $] 4$ & 4305.890 & 0.173 & 3.179 & [FeII $] 21$ & 4583.829 & 1.000 & 8.397 & FeII 38 \\
\hline 5006.624 & 0.081 & 1.591 & [FeII] 4 & 4346.852 & 0.166 & 4.051 & [FeII $] 21$ & 4620.513 & 0.080 & 0.506 & FeII 38 \\
\hline \multirow[t]{2}{*}{5035.484} & 0.011 & 0.084 & [FeII] 4 & 4352.778 & 0.266 & 5.576 & [FeII] 21 & & & & \\
\hline & & & & 4358.471 & 0.191 & 3.341 & {$[\mathrm{FeII}] 21^{h}$} & 4923.921 & 0.902 & 6.413 & FeII 42 \\
\hline 4358.095 & 0.244 & 3.341 & {$[\mathrm{FeII}] 6^{c}$} & 4372.427 & 0.208 & 3.484 & [FeII] 21 & 5018.434 & 0.967 & 13.70 & FeII 42 \\
\hline 4382.742 & 0.115 & 1.550 & {$[\mathrm{FeII}] 6$} & & & & & 5169.030 & 1.000 & 14.08 & FeII 42 \\
\hline 4416.266 & 1.000 & 15.47 & [FeII $] 6$ & 4017.378 & 0.751 & 0.312 & [FeII] 24 & & & & \\
\hline 4432.447 & 0.074 & 1.413 & [FeII $] 6$ & 4033.972 & 1.000 & 0.456 & [FeII $] 24$ & 4656.974 & 0.407 & 0.445 & FeII 43 \\
\hline 4457.945 & 0.550 & 7.050 & [FeII $] 6$ & 4079.989 & 0.791 & 0.286 & [FeII] 24 & 4731.439 & 1.000 & 0.717 & FeII 43 \\
\hline 4470.294 & 0.024 & 0.482 & {$[\mathrm{FeII}] 6$} & 4084.320 & 0.393 & 0.166 & [FeII $] 24$ & & & & \\
\hline 4492.634 & 0.158 & 2.078 & [FeII] 6 & 5083.731 & 0.088 & 0.5085 & [FeII] 35 & 5234.620 & 0.315 & 6.277 & FeII 49 \\
\hline 4509.602 & 0.079 & 1.132 & [FeII] 6 & 5163.951 & 1.000 & 3.814 & [FeII] 35 & 5275.994 & 0.381 & 3.344 & FeII 49 \\
\hline 4528.384 & 0.074 & 0.949 & [FeII] 6 & 5199.173 & 0.385 & 3.604 & [FeII] 35 & 5316.609 & 1.000 & 8.765 & FeII 49 \\
\hline \multirow[t]{2}{*}{4533.003} & 0.033 & 0.423 & [FeII $] 6$ & & & & & 5325.553 & 0.035 & 0.380 & FeII 49 \\
\hline & & & & 4266.349 & 0.176 & 0.161 & [FeII] 36 & 5347.228 & 0.118 & 0.969 & FeII 49 \\
\hline 4287.394 & 1.000 & 25.78 & [FeII] 7 & 4329.430 & 0.111 & 0.106 & [FeII $] 36$ & 5425.257 & 0.034 & 0.204 & FeII 49 \\
\hline 4359.333 & 0.777 & 18.58 & [FeII] 7 & 4384.210 & 1.000 & 0.850 & [FeII] 36 & 5476.989 & 0.134 & 2.763 & FeII 49 \\
\hline 4413.781 & 0.551 & 13.68 & [FeII] 7 & 4402.560 & 0.161 & 0.131 & [FeII] 36 & & & & \\
\hline 4452.098 & 0.369 & 7.827 & [FeII] 7 & 4439.710 & 0.196 & 0.219 & [FeII] 36 & 4577.791 & 0.133 & 0.101 & FeII 54 \\
\hline \multirow{2}{*}{4474.904} & 0.162 & 4.585 & {$[\mathrm{FeII}] 7$} & & & & & 4627.866 & 1.000 & 0.897 & FeII 54 \\
\hline & & & & 4327.039 & 0.255 & 0.111 & FeII 20 & & & & \\
\hline 5412.654 & 0.756 & 2.077 & [FeII $] 17$ & 4399.858 & 1.000 & 0.259 & FeII 20 & 6147.735 & 0.051 & 0.237 & FeII 74 \\
\hline 5495.824 & 0.465 & 2.337 & {$[\mathrm{FeII}] 17$} & 4558.572 & 0.840 & 0.296 & FeII 20 & 6149.238 & 0.076 & 0.505 & FeII 74 \\
\hline \multirow[t]{2}{*}{5527.339} & 1.000 & 2.532 & [FeII] 17 & & & & & 6238.375 & 0.151 & 0.773 & FeII 74 \\
\hline & & & & 4124.793 & 1.000 & 0.253 & FeII 22 & 6239.953 & 0.040 & 0.186 & FeII 74 \\
\hline 5107.942 & 0.066 & 0.733 & [FeII] 18 & 4205.473 & 0.533 & 0.131 & FeII 22 & 6247.562 & 0.325 & 0.887 & FeII 74 \\
\hline 5158.001 & 0.491 & 4.213 & {$[$ FeII $] 18^{e}$} & & & & & 6416.905 & 0.129 & 0.119 & FeII 74 \\
\hline 5181.948 & 0.195 & 3.849 & [FeII] 18 & 4128.735 & 0.038 & 0.328 & FeII 27 & 6456.376 & 1.000 & 0.633 & FeII 74 \\
\hline 5268.875 & 0.334 & 2.358 & [FeII $] 18$ & 4173.450 & 0.268 & 2.285 & FeII 27 & & & & \\
\hline \multirow[t]{2}{*}{5433.129} & 0.339 & 1.584 & [FeII $] 18$ & 4303.166 & 0.233 & 1.701 & FeII 27 & & & & \\
\hline & & & & 4351.764 & 0.538 & 4.524 & FeII 27 & 4068.600 & 1.000 & 4.199 & [SII] 1 \\
\hline 5072.392 & 0.037 & 0.255 & [FeII] 19 & 4385.381 & 0.260 & 1.802 & FeII 27 & 4076.349 & 0.166 & 0.730 & [SII] 1 \\
\hline 5111.627 & 0.113 & 0.820 & [FeII] 19 & & & & & & & & \\
\hline 5158.777 & 0.613 & 4.213 & [FeII] $19^{g}$ & 4122.638 & 0.157 & 0.913 & FeII 28 & 4290.222 & 0.491 & 0.180 & TiII 41 \\
\hline 5220.059 & 0.298 & 1.722 & [FeII] 19 & 4178.855 & 1.000 & 5.632 & FeII 28 & 4300.052 & 1.000 & 0.362 & TiII 41 \\
\hline 5261.621 & 1.000 & 5.186 & [FeII $] 19$ & 4258.155 & 0.158 & 0.806 & FeII 28 & 4307.900 & 0.644 & 0.276 & TiII 41 \\
\hline 5296.829 & 0.140 & 0.697 & [FeII $] 19$ & 4296.567 & 0.365 & 1.792 & FeII 28 & 4312.861 & 0.333 & 0.114 & TiII 41 \\
\hline 5333.646 & 0.919 & 3.845 & [FeII $] 19$ & 4369.404 & 0.092 & 0.414 & FeII 28 & 4314.979 & 0.231 & 0.082 & TiII 41 \\
\hline \multirow[t]{2}{*}{5376.452} & 0.771 & 2.909 & [FeII $] 19$ & & & & & & & & \\
\hline & & & & 4278.13 & 1.000 & 0.942 & FeII 32 & 4201.171 & 1.000 & 2.466 & [NiII] 3 \\
\hline 4774.718 & 0.296 & 6.730 & [FeII $] 20$ & 4314.29 & 0.853 & 0.759 & FeII 32 & 4326.236 & 0.942 & 2.348 & [NiII] 3 \\
\hline 4814.534 & 1.000 & 16.61 & [FeII 20 & 4338.70 & 0.163 & 0.232 & FeII 32 & 4485.212 & 0.401 & 0.879 & [NiII] 3 \\
\hline 4852.730 & 0.019 & 0.565 & [FeII 20 & & & & & 4628.046 & 0.351 & 0.956 & [NiII] 3 \\
\hline 4874.485 & 0.346 & 5.183 & [FeII 20 & 4472.921 & 0.054 & 0.595 & FeII 37 & & & & \\
\hline 4905.339 & 0.531 & 4.127 & [FeII $] 20$ & 4489.185 & 0.286 & 2.063 & FeII $37^{i}$ & 3993.059 & 1.000 & 3.779 & {$[\mathrm{NiII}] 4$} \\
\hline 4947.373 & 0.129 & 0.562 & [FeII] 20 & 4491.401 & 0.320 & 2.410 & FeII 37 & 4033.038 & 0.423 & 1.782 & {$[\mathrm{NiII}] 4$} \\
\hline 4950.744 & 0.183 & 0.761 & [FeII] 20 & 4515.337 & 0.687 & 5.751 & FeII 37 & 4248.799 & 0.447 & 1.354 & {$[\mathrm{NiII}] 4$} \\
\hline 4973.388 & 0.306 & 6.099 & [FeII] 20 & 4520.225 & 0.484 & 4.343 & FeII 37 & 4285.306 & 0.009 & 0.021 & [NiII] 4 \\
\hline 5005.512 & 0.386 & 6.703 & [FeII] 20 & 4534.166 & 0.118 & 0.930 & FeII 37 & 4294.092 & 0.450 & 1.410 & [NiII] 4 \\
\hline 5020.233 & 0.310 & 3.967 & [FeII] 20 & 4555.890 & 0.685 & 5.172 & FeII 37 & & & & \\
\hline \multirow[t]{4}{*}{5043.520} & 0.220 & 1.366 & [FeII $] 20$ & 4582.835 & 0.155 & 1.374 & FeII 37 & 4147.238 & 1.000 & 0.700 & [NiII] 10 \\
\hline & & & & 4629.336 & 1.000 & 9.045 & FeII 37 & 4310.341 & 0.180 & 0.118 & {$[\mathrm{NiII}] 10$} \\
\hline & & & & 4666.750 & 0.202 & 1.817 & FeII 37 & 4461.464 & 0.780 & 0.441 & [NiII] 10 \\
\hline & & & & & & & & 4573.357 & 0.247 & 0.100 & {$[\mathrm{NiII}] 10$} \\
\hline
\end{tabular}

$a$ : blend of $4664.440,4665.645 \AA$ lines of this same multiplet

$b$ : blend of $4798.274,4799.293 \AA$ lines of this same multiplet

c: unresolved blend with [Fe II] 21 4358.471. Flux split half-and-half between the two lines.

$d$ : unresolved blend with Fe II 37 4489.183. Flux split half-and-half between the two lines.

$e$ : unresolved blend with [Fe II] 19 5158.777. Flux split half-and-half between the two lines.

$f$ : unresolved blend with Fe II 495347.228 . Flux split half-and-half between the two lines.

$g$ : unresolved blend with [Fe II] 18 5158.001. Flux split half-and-half between the two lines.

$h$ : unresolved blend with [FeII] 6 4358.095. Flux split half-and-half between the two lines.

$i$ : unresolved blend with [FeII] 64488.749 . Flux split half-and-half between the two lines. 